\title{
Functional significance of M-type potassium channels in nociceptive cutaneous sensory endings
}

\section{Gayle M. Passmore ${ }^{1 *}$, Joanne M. Reilly ${ }^{1}$, Matthew Thakur ${ }^{1}$, Vanessa N. Keasberry ${ }^{1,2}$, Stephen J. Marsh ${ }^{1}$, Anthony H. Dickenson ${ }^{1}$ and David A. Brown ${ }^{1}$}

${ }^{1}$ Department of Neuroscience, Physiology and Pharmacology, University College London, London, UK

2 Department of Cell Physiology and Pharmacology, University of Leicester, Leicester, UK

\section{Edited by:}

Nikita Gamper, University of

Leeds, UK

Reviewed by:

Nikita Gamper, University of

Leeds, UK

Doug Krafte, Pfizer, USA

*Correspondence:

Gayle M. Passmore, Department of

Neuroscience, Physiology and

Pharmacology, University College

London, Gower Street, London

WC1E 6BT, UK

e-mail: gayle.passmore@ucl.ac.uk
M-channels carry slowly activating potassium currents that regulate excitability in a variety of central and peripheral neurons. Functional M-channels and their Kv7 channel correlates are expressed throughout the somatosensory nervous system where they may play an important role in controlling sensory nerve activity. Here we show that Kv7.2 immunoreactivity is expressed in the peripheral terminals of nociceptive primary afferents. Electrophysiological recordings from single afferents in vitro showed that block of M-channels by $3 \mu \mathrm{M}$ XE991 sensitized A $\delta$ - but not C-fibers to noxious heat stimulation and induced spontaneous, ongoing activity at $32^{\circ} \mathrm{C}$ in many $A \delta$-fibers. These observations were extended in vivo: intraplantar injection of XE991 selectively enhanced the response of deep dorsal horn $(\mathrm{DH})$ neurons to peripheral mid-range mechanical and higher range thermal stimuli, consistent with a selective effect on A $\delta$-fiber peripheral terminals. These results demonstrate an important physiological role of $\mathrm{M}$-channels in controlling nociceptive $A \delta$-fiber responses and provide a rationale for the nocifensive behaviors that arise following intraplantar injection of the M-channel blocker XE991.

Keywords: M-channel, Kv7, KCNQ, excitability, XE991, retigabine

\section{INTRODUCTION}

The M-channel is a key regulator of excitability in a variety of central and peripheral neurons (Brown and Passmore, 2009). At membrane potentials sub-threshold to action potential initiation slow activation of the M-current exerts a membrane potentialclamping effect and limits the ability of a neuron to fire repetitively (Brown, 1988). When M-channels are inhibited, either following activation of a variety of G-protein coupled receptors (see for example Brown and Constanti, 1980; Adams et al., 1982) or by direct channel block with compounds such as linopirdine and XE991 (Aiken et al., 1995; Wang et al., 1998; Zaika et al., 2006), this potential-clamping effect is removed and tonic firing may be readily induced. Whilst this phenomenon has been clearly demonstrated in sympathetic (Wang et al., 1998; Zaika et al., 2006), parasympathetic (Cuevas et al., 1997), and some central (e.g., Peters et al., 2005; Shen et al., 2005) neurons, the physiological importance of $\mathrm{M}$-current activation in other neurons is less clear.

Functional M-channels and their Kv7 channel correlates have been detected at several loci in the somatosensory pathway including dorsal root ganglion (DRG) and dorsal horn (DH) neurons (Passmore et al., 2003; Rivera-Arconada and LopezGarcia, 2005), peripheral nerve axons (Schwarz et al., 2006; Lang et al., 2008; Sittl et al., 2010), and the peripheral and central terminals of primary afferents (Rivera-Arconada and Lopez-Garcia, 2006; Roza and Lopez-Garcia, 2008; Heidenreich et al., 2011). Whilst positive modulation of these channels by the Kv7 current enhancer retigabine produces membrane hyperpolarization and reduces excitability (Passmore et al., 2003;
Rivera-Arconada and Lopez-Garcia, 2005, 2006; Lang et al., 2008; Roza and Lopez-Garcia, 2008), the evidence for a physiologically important role from, for example, the effects of selective channel block with low concentrations of XE991 is more limited. Thus, linopirdine and XE991 produced relatively modest effects on membrane potential and excitability in somatosensory neurons in DRG (Passmore et al., 2003; Liu et al., 2010; Mucha et al., 2010), DH (Rivera-Arconada and Lopez-Garcia, 2005) and nodose ganglion (Wladyka and Kunze, 2006; Wladyka et al., 2008).

It is possible that M-channels play a more important role at the peripheral rather than the central terminals of sensory fibers, and indeed intraplantar injection of XE991 into the hindpaw produced acute pain in rats (Linley et al., 2008). The latter suggests that M-channels, located in cutaneous sensory endings, are important in controlling the responses of nociceptive primary afferents. We have tested this possibility using the in vitro skinsaphenous nerve preparation and in vivo wide dynamic range (WDR) neuron recording. We have examined the effect of local application of the M-channel blocker XE991 on the excitability of primary afferent fibers and the effect of intraplantar injections of XE991 on the electrical, mechanical, and thermal-evoked responses of DH neurons. We have also examined the peripheral distribution of one of the principal subunits of the M-channel (Kv7.2: Wang et al., 1998) using immunofluorescence. Some parts of this work have previously been published in abstract or review form (Passmore and Brown, 2007; Reilly et al., 2008; Brown and Passmore, 2009). 


\section{METHODS \\ ETHICAL APPROVAL}

This research was approved by the UCL Ethical Review Panel and undertaken in conformity with the requirements of the UK Home Office Animals (Scientific Procedures) Act of 1986.

\section{IMMUNOFLUORESCENCE}

Sprague-Dawley rats weighing approximately $150 \mathrm{~g}$ were deeply anaesthetized with ketamine/xylazine hydrochloride solution $(1 \mathrm{ml} / \mathrm{kg}$, i.p., Sigma, UK) and transcardially perfused with icecold heparinized saline [9\% w/V NaCl (VWR, UK), 50,000 U/l Heparin (Sigma, UK)], then 4\% paraformaldehyde (EMS Diasum, USA) in phosphate-buffered saline (PBS; Sigma, UK). The hairy skin from the dorsal surface of the hind paw was removed, post fixed for $3 \mathrm{~h}$ with $4 \%$ paraformaldehyde in PBS, rinsed with PBS, and then cryoprotected via immersion overnight in a $30 \%$ sucrose (VWR, UK)/0.01\% NaAzide (Sigma, UK)/PBS solution.

The skin was sliced into $40 \mu \mathrm{m}$ cryosections using a freezing microtome (Leica Microsystems, Germany) and free floating sections were placed into PBS. The sections were incubated with blocking solution [PBS containing 3\% goat serum and 3\% Triton ${ }^{\circledR}$ X-100 (Sigma, UK)] then incubated overnight at $4^{\circ} \mathrm{C}$ with primary antibody. Skin sections were co-stained with antibodies against Kv7.2 (1:100, Neuromabs, USA) and either TRPV1 (1:1000, Abcam, UK), neurofilament H (1:1000, Neuromics, USA), peripherin (1:1000, Abcam, UK), or tyrosine hydroxylase, (1:1000, Abcam, UK). The sections were rinsed and the Kv7.2 antibody was incubated with a biotinylated secondary antibody (Sigma, UK) then Cy3 conjugated streptavidin (Sigma, UK). All other primary antibodies were detected via fluorescein (FITC)-conjugated secondary antibodies (Sigma, UK). Staining was visualized using a Leica CA TCS SP2 AOBS spectral confocal microscope (Leica, Germany). The gain, exposure time, and wavelength were optimized for the excitation and emission spectra of each fluorophor. Control experiments performed without primary antibodies did not show labelling above background.

\section{SKIN-SAPHENOUS NERVE PREPARATION}

The in vitro rat skin-saphenous nerve preparation was used to record single nerve fiber activity and has been described in detail elsewhere (Reeh, 1986; Zimmermann et al., 2009).

Sprague-Dawley rats (150-200 g) were killed by $\mathrm{CO}_{2}$ asphyxiation followed by cervical dislocation. The hairy skin from the dorsal surface of the hind paw was excised together with the saphenous nerve trunk, mounted corium side-up in an organ bath and continuously superfused $\left(16 \mathrm{ml} \mathrm{min}^{-1}\right)$ with an oxygenated modified synthetic interstitial fluid (SIF) containing (in $\mathrm{mM}$ ): $\mathrm{NaCl}, 139 ; \mathrm{NaHCO}_{3}, 21$; Glucose, $10 ; \mathrm{NaH}_{2} \mathrm{PO}_{4}, 0.6$; $\mathrm{KCl}, 3.5 ; \mathrm{MgCl}_{2}, 1 ; \mathrm{CaCl}_{2}, 1.3$ at $\mathrm{pH} 7.4$ and a temperature of approximately $32^{\circ} \mathrm{C}$.

The saphenous nerve bundle was threaded through a hole into a recording chamber containing a shallow layer of SIF where it was de-sheathed by peeling back the epineurium. The hole between the two chambers was then filled with grease and the nerve bundle overlaid with paraffin oil and teased into small filaments until single unit activity could be resolved. Single unit activity was initially determined following electrical stimulation of the nerve trunk using an isolated constant voltage stimulator (1 ms duration; Digitimer Ltd., UK) whilst the receptive fields of single fibers were identified following manual probing of the skin with a blunt glass rod.

Fibers were classified according to their conduction velocity, von Frey threshold, and response to suprathreshold force (Zimmermann et al., 2009). Conduction velocity was determined following stimulation of the receptive field using a Teflon-coated steel electrode (Linton Instruments, UK). Fibers conducting below $1.2 \mathrm{~ms}^{-1}$ were classed as unmyelinated C-fibers and those conducting between $1.2 \mathrm{~ms}^{-1}$ and $15.0 \mathrm{~ms}^{-1}$ as thinly myelinated A $\delta$-fibers.

The von Frey threshold was determined using a series of calibrated von Frey hairs with an uniform tip diameter of $0.8 \mathrm{~mm}$ (Zimmermann et al., 2009) and was taken as the minimum force required to elicit three or more action potentials.

Drugs were applied directly to the receptive fields of identified peripheral primary afferents by continuous perfusion

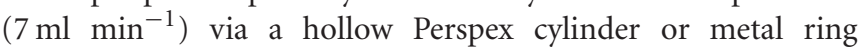
(Zimmermann et al., 2009). Heat ramps were generated using a Marlow temperature controller and Peltier device or by a radiant heat lamp as previously described (Zimmermann et al., 2009) with an interval of $5 \mathrm{~min}$ between successive heat ramps to limit sensitization of the response (Du et al., 2001).

Single fiber activity was recorded using platinum wire electrodes (Harvard Apparatus, UK) and a low-noise AC-coupled amplifier (Neurolog, Digitimer Ltd., UK), monitored continuously using a loudspeaker and oscilloscope and digitized using a CED 1401 plus interface (Cambridge Electronic Design). Data were filtered using a band-pass filter with a bandwidth between $100 \mathrm{~Hz}$ and $1.5 \mathrm{kHz}$ and sampled between 12 and $20 \mathrm{kHz}$. Data were analyzed offline using Spike2 software (Cambridge Electronic Design).

Data are presented as mean \pm SEM for all values. Statistical comparisons between pre- and post-drug responses were performed using One-Way ANOVA followed by Bonferroni's multiple comparison post-test.

\section{VAGUS NERVE RECORDING}

Rat vagus nerves were isolated, desheathed, and the intact nerve placed in a three-chambered bath with the partitions grease-sealed, as previously described (Marsh et al., 1987). The nerve was electrically stimulated via platinum electrodes placed in the proximal chamber. Electrical potentials were recorded from the central chamber with respect to the distal chamber using $\mathrm{Ag} / \mathrm{AgCl}$ electrodes with $\mathrm{DC}$ amplification. Procaine $(1 \mathrm{mM})$ and tetrodotoxin (TTX, $0.5 \mu \mathrm{M})$ were added to the distal chamber to block distal action potentials and convert recorded compound action potentials to monopolar form. Drugs were continuously perfused via the central chamber at a rate of $4 \mathrm{ml} \mathrm{min}-1$. Experiments were performed at $20^{\circ} \mathrm{C}$ to minimize noise and DC drift. Data were filtered and sampled at 10 and $100 \mathrm{kHz}$, respectively, and are presented as mean \pm SEM for all values. Statistical comparisons were performed using paired Student's $t$-test. 


\section{In vivo SPINAL CORD ELECTROPHYSIOLOGY}

In vivo electrophysiological studies were performed as previously described (Urch and Dickenson, 2003). Briefly, animals were anaesthetized and maintained for the duration of the experiment with isofluorane (1.5-1.7\%) delivered in a gaseous mix of $\mathrm{N}_{2} \mathrm{O}$ $(66 \%)$ and $\mathrm{O}_{2}(33 \%)$. A laminectomy was performed to expose the L4- 5 segments of the spinal cord. Extracellular recordings were made from ipsilateral deep DH neurons (laminae V-VI) using parylene coated tungsten electrodes (A-M Systems, USA). The neurons included in this study met the following criteria: they had a receptive field on the plantar hindpaw; they all responded with at least 50 spikes to both light touch ( $8 \mathrm{~g}$ von frey) and noxious inputs (60 g von Frey and $48^{\circ} \mathrm{C}$ heat); they responded to natural stimuli in a graded manner with coding of increasing intensity; they exhibited windup when repeatedly stimulated; and they were situated at a depth of $>500 \mu \mathrm{M}$ from the surface of the spinal cord.

A train of 16 transcutaneous electrical stimuli $(2 \mathrm{~ms}$ wide pulses, $0.5 \mathrm{~Hz}$ ) applied at three times the threshold current for C-fiber activation of the $\mathrm{DH}$ cell was delivered via stimulating electrodes inserted into the peripheral receptive field in the hindpaw. A post-stimulus time histogram (PSTH) was constructed such that responses evoked by $\mathrm{A} \beta-(0-20 \mathrm{~ms})$, A $\delta-$ (20-90 ms) and C-fibers (90-350 ms) were separated and quantified on the basis of latency. Responses occurring after the C-fiber latency band were taken to be the post-discharge of the cell (350-800 ms).

The center of the peripheral receptive field was also stimulated using punctate mechanical and thermal stimuli (von Frey filaments, 2, 6, 8, 15, 26, and $60 \mathrm{~g}$ and a water jet applied at, 35, 40, 45 , and $\left.48^{\circ} \mathrm{C}\right)$. Application of each von Frey hair was separated by a minimum interval period of $50 \mathrm{~s}$. All natural stimuli were applied for a period of $10 \mathrm{~s}$ per stimulus. Data was captured and analyzed by a CED 1401 interface coupled to a computer running Spike 2 software (Cambridge Electronic Design; PSTH and rate functions).

Pharmacological assessment was carried out on one ipsilateral neuron only per animal. One round of testing was performed every $20 \mathrm{~min}$, and consisted of a train of electrical stimuli followed by graded natural stimuli as described above. Following three consecutive stable control trials $(<10 \%$ variation for the C-fiber-evoked response) neuronal responses were averaged to give the pre-drug control values. XE991 (Tocris Bioscience, UK) was dissolved in $0.9 \%$ saline solution at a concentration of $200 \mu \mathrm{M}$, and administered via intraplantar injection close to the hindpaw receptive field. This concentration has previously been shown to induce nociceptive behavior (Linley et al., 2008). Intraplantar saline alone does not alter responses in this preparation (Elmes et al., 2004). The effect of the drug was followed for an hour, with tests carried out at 10, 30, and $50 \mathrm{~min}$. The value of greatest change from the baseline for each response (electrical stimuli, natural stimuli) was then found. Two-Way ANOVA with Bonferroni's multiple comparison post-test was used to compare pre-dose baselines with greatest change from baseline values for each natural response, while paired Student's $t$-test was used to compare pre-and post-dose electrically evoked responses.

\section{RESULTS}

\section{Kv7.2 IS EXPRESSED IN NOCICEPTIVE SENSORY ENDINGS}

Since Kv7.2 is present in small diameter DRG neurons, and functional M-currents can be recorded from DRG neurons in both culture and slice preparations (Passmore et al., 2003; Rose et al., 2011), we hypothesized that it might also be expressed at cutaneous sensory endings.

We examined Kv7.2 expression in cryosections from rat hairy skin. Kv7.2 was detected in both myelinated and unmyelinated nerve bundles (Figure 1, panels A-F), as indicated by costaining for neurofilament $\mathrm{H}$ and peripherin, respectively. Some Kv7.2 positive fibers also expressed TRPV1 (Figure 1, panels G-I), a marker of nociceptive nerve bundles. Kv7.2 also colocalized with tyrosine hydroxylase (Figure 1, panels J-L) indicating expression in sympathetic fibers as expected (Hadley et al., 2003).

Interestingly, not all fibers of each of these fiber classes stained for Kv7.2, witness the neurofilament $\mathrm{H}$-positive/Kv7.2-negative bundle in panel $\mathbf{C}$ and the peripherin-positive/Kv7.2-negative fibers within the fiber bundle in panel $\mathbf{F}$.
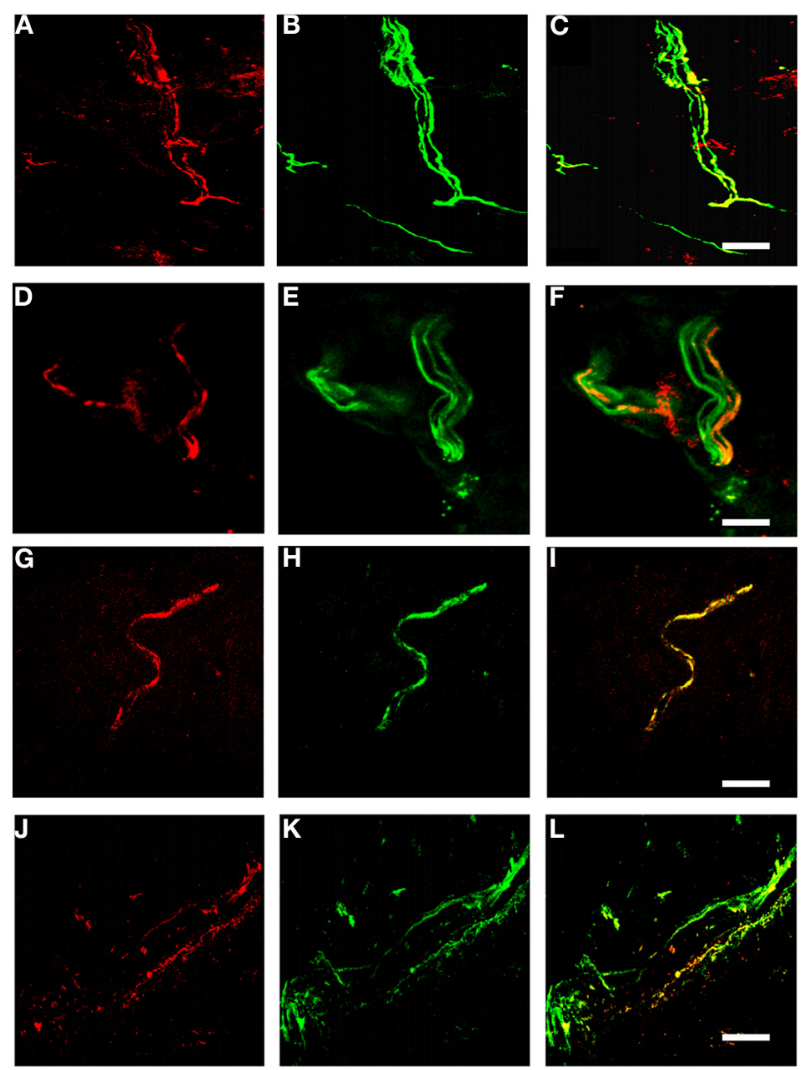

FIGURE 1 | Kv7.2 in rat cutaneous sensory endings. (A-L) Rat hairy skin sections stained with antibodies against Kv7.2 (red). Pictures show Kv7.2 localization in myelinated fibers (A-C, counterstained for neurofilament $\mathbf{H}$ ), unmyelinated fibers (D-F, counterstained for peripherin), nociceptive fibers (G-I, counterstained for TRPV1), and sympathetic fibers

( $\mathbf{J}-\mathbf{L}$, counterstained for tyrosine hydroxylase). Scale bar, $20 \mu \mathrm{M}$ in panels $\mathbf{A}-\mathbf{C}, \mathbf{G}-\mathbf{I}$, and $\mathbf{J}-\mathbf{L}, 10 \mu \mathrm{M}$ in panels $\mathbf{D}-\mathbf{F}$ 


\section{NOCICEPTIVE SENSORY ENDINGS EXPRESS FUNCTIONAL M-CHANNELS}

To determine whether functional M-channels are present at nociceptive sensory nerve endings in the skin we used an in vitro skin-saphenous nerve preparation to examine the single unit responses of identified $\mathrm{A} \delta$ - and $\mathrm{C}$-fibers to noxious heat stimulation under control conditions and in the presence of the M-channel modulators retigabine and XE991.

Two or more (up to four) control heat ramps were applied prior to drug application to determine whether any sensitization/desensitization of the response occurred from repeated heat stimulation. Drugs were applied once the response began to stabilize.

Figure 2 shows an example of the experimental approach used. The response of a mechano-heat A $\delta$-fiber (AMH) is shown from which it is clear that the number of action potentials elicited in response to a heat ramp from $30^{\circ} \mathrm{C}$ to $44^{\circ} \mathrm{C}$ (top panels in Figure 2A-F) was altered following exposure of the receptive field to the M-channel modulators retigabine and XE991, respectively. Under control conditions the heat ramp induced a robust firing response (Figure 2A), which was inhibited following exposure of the receptive field to $10 \mu \mathrm{M}$ retigabine for $5 \mathrm{~min}$ (Figure 2B), but recovered during washing (Figures $2 \mathrm{C}$ and D). Application of XE991 $(3 \mu \mathrm{M})$ to the receptive field produced a marked sensitization to the subsequent heat stimulation and induced spontaneous firing at $30^{\circ} \mathrm{C}$ (Figure 2E). After $20 \mathrm{~min}$ of washing, the response recovered toward that seen in control (Figure 2F).

\section{M-CHANNEL OPENING DAMPENS A $\delta$ - AND C-FIBER RESPONSES TO NOXIOUS HEAT}

We examined the effect of retigabine on the responses of a further six AMH-fiber responses. Five minutes' exposure of the receptive fields to $10 \mu \mathrm{M}$ retigabine produced a significant $(P<0.005)$ inhibition of AMH-fiber responses to noxious heat (Figure 3A). It reduced the mean number of action potentials per heat stimulation from $58.6 \pm 15.8$ and $52.3 \pm 13.3$ in two control ramps, respectively, to $21.7 \pm 12.9$ at $5 \mathrm{~min}$ and to $7.3 \pm 3.6$ at $10 \mathrm{~min}$ after application. The effect of retigabine was relatively persistent and irreversible over $15 \mathrm{~min}$ in four of the seven fibers; the other three fibers began to recover after 10-15 min washing, the mean number of action potentials per heat stimulation for all seven fibers increasing to $9.1 \pm 6.1$ and $15.5 \pm 10.8$, respectively. In spite of this persistent reduction in activity, fibers could still either be electrically stimulated or responded to noxious heat following exposure of their receptive fields to $3 \mu \mathrm{M}$ XE991, as in Figure 2.

In contrast, retigabine had much less effect on C-mechanoheat $(\mathrm{CMH}) / \mathrm{C}$-mechano-cold-heat $(\mathrm{CMCH})$ fiber responses to noxious heat. A pooled time-plot (Figure 3B) did not reveal a significant reduction in response $(n=10 ; P>0.05)$ as determined by ANOVA followed by Bonferroni's or Tukey's multiple comparison post-tests; the mean number of action potentials per heat stimulation were $39.8 \pm 7.6$ and $36.8 \pm$ 7.2 in two control ramps, respectively, $26.6 \pm 8.8$ at $5 \mathrm{~min}$ and $23.0 \pm 6.8$ at $10 \mathrm{~min}$ after retigabine application, and

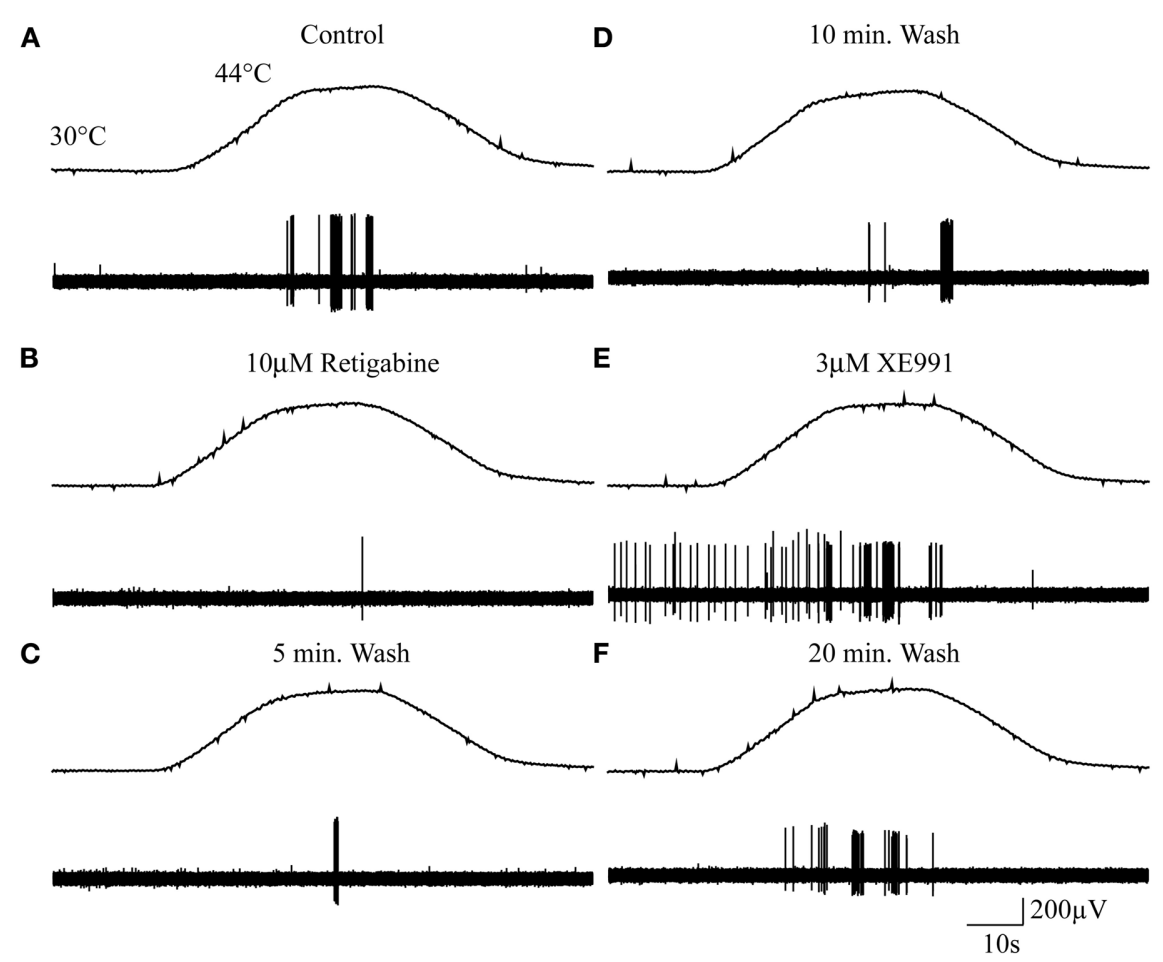

FIGURE 2 | Cutaneous sensory endings express functional M-channels. (A-F) Example of a mechano-heat (AMH) A $\delta$-fiber response to noxious heat stimulation pre- and post-exposure to the M-channel modulators retigabine and XE991. Top panels in A-F show the noxious heat ramps that ramp from $30^{\circ} \mathrm{C}$ to $44^{\circ} \mathrm{C}$. Control response (A), $10 \mu \mathrm{M}$ retigabine (B), 5 min wash (C), 10 min wash (D), $3 \mu \mathrm{M}$ XE991 (E), and 20 min wash (F). (The apparent decline in action potential amplitude from $\mathbf{A}$ through $\mathbf{F}$ probably results from drying-out of the nerve following prolonged immersion in liquid paraffin.) 


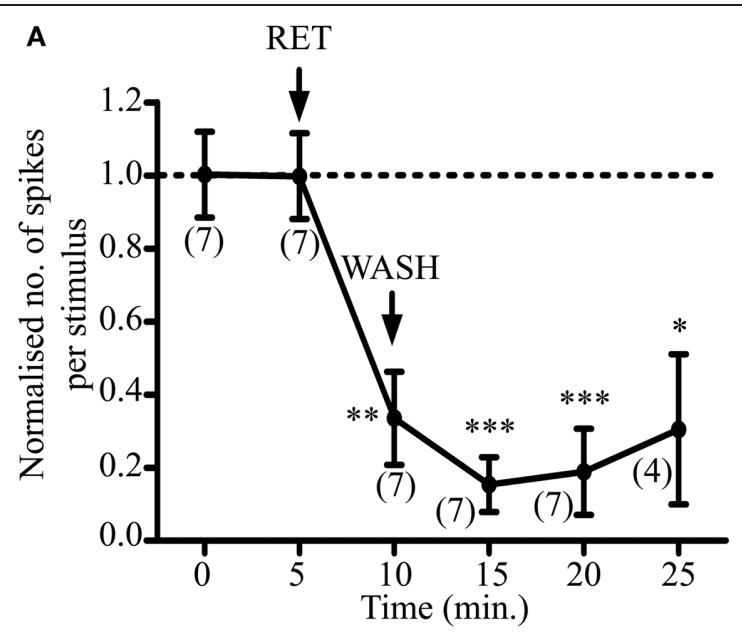

B

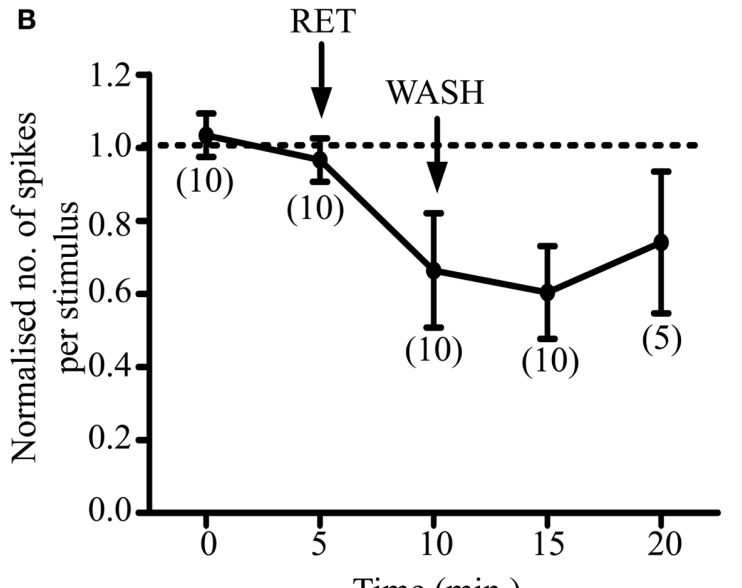

C

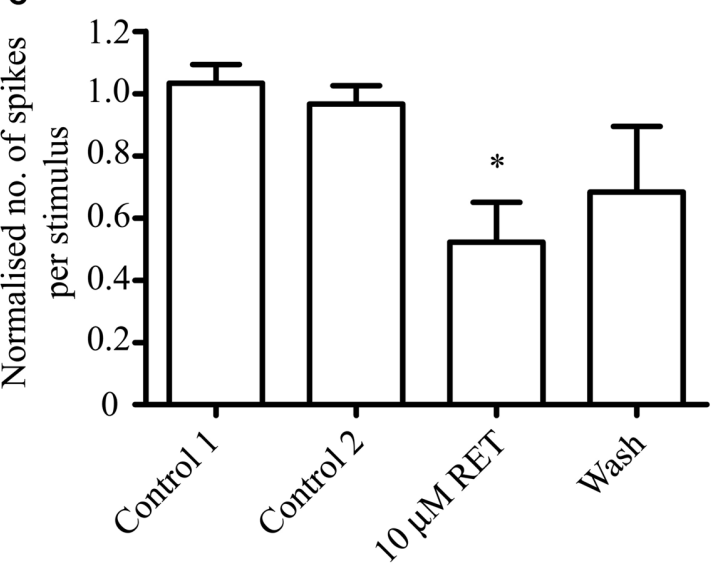

FIGURE 3 | Opening M-channels in sensory endings with retigabine (RET) strongly dampens AMH-fiber responses to noxious heat but has less effect on C-fibers. Normalized responses to noxious heat in AMH-fibers (A) and C-fibers (B and $\mathbf{C}$ ). Normalization is to the mean of the two control responses prior to retigabine exposure. $\mathrm{AMH}$-fiber responses were significantly inhibited by $10 \mu \mathrm{M}$ retigabine ${ }^{*} P<0.05,{ }^{*} P<0.01$, *** $P<0.005$ ). (C) $C$-fiber minimal responses to heat measured at either 5 or $10 \mathrm{~min}$ following exposure to retigabine were significantly reduced $\left.{ }^{*} P<0.05\right)$ compared to control. Number of fibers shown in brackets. $\mathrm{AMH}$ - and C-fiber mean conduction velocities were $3.98 \pm 0.99 \mathrm{~ms}^{-1}$ $(n=7)$ and $0.65 \pm 0.09 \mathrm{~ms}^{-1}(n=10)$, respectively.
$32.6 \pm 13.7$ after $10 \mathrm{~min}$ washing. However, to some extent this apparently-weak effect may have been due to variations in the time course of retigabine action on individual Cfibers, since the minimal response of the fibers after retigabine application (measured at either time-point within the $10 \mathrm{~min}$ period after application) was significantly reduced to $20.8 \pm 7.2$ action potentials per heat stimulation $(P<0.05$; Figure 3C). Notwithstanding, the effect of retigabine on the $\mathrm{C}$ fiber responses was clearly smaller and less convincing than that on A $\delta$ fibers.

\section{M-CHANNELS REGULATE A $\delta$-FIBER BUT NOT NOCICEPTIVE C-FIBER RESPONSES TO NOXIOUS HEAT}

The marked sensitization to heat produced by XE991 (Figure 2E) suggests that $\mathrm{M}$-channels may play a pivotal role in the physiological regulation of action potential initiation at the primary afferent ending. We examined this further by testing the effects of XE991 on the responses of $32 \mathrm{~A} \delta$ - and $8 \mathrm{C}$-fibers to noxious heat.

The most striking effect was the sensitization of "apparent" heat insensitive $\mathrm{A} \delta$-mechano (AM) fibers to heat (Figures $4 \mathrm{~A}-\mathrm{C}$ ). Five minutes' exposure of the receptive fields of 15 AM-fibers to $3 \mu \mathrm{M}$ XE991 increased the mean number of action potentials per heat stimulation from $0.5 \pm 0.3$ and $0.2 \pm 0.1$ in two controls, respectively, to $130.4 \pm 50.2$ at $5 \mathrm{~min}$ after application. The effect of XE991 was persistent, the mean number of action potentials increasing to $201.9 \pm 82.0$ and $276.4 \pm 111$ at 10 and $15 \mathrm{~min}$ after application, respectively. After $20 \mathrm{~min}$ washing the mean number of action potentials reduced to $84.9 \pm 31.1$ per heat stimulation. As illustrated in Figure 2E, 10 of the 15 fibers exhibited spontaneous activity at $30-32^{\circ} \mathrm{C}$ following exposure of their receptive fields to $3 \mu \mathrm{M}$ XE991. Ongoing activity at $32^{\circ} \mathrm{C}$ in the absence of a response to noxious heat was also seen in an additional AM-fiber following exposure of its receptive field to $3 \mu \mathrm{M}$ XE991. In contrast, 6 AM-fibers (out of a total of 32 sampled) were insensitive to XE991; exposure of their receptive fields to $3 \mu \mathrm{M}$ XE991 failed to sensitize these fibers to noxious heat and no ongoing activity at $32^{\circ} \mathrm{C}$ was observed. However, these fibers developed responses to noxious heat following exposure of their receptive fields to $20 \mathrm{mM} \mathrm{KCl}$ (data not shown).

XE991 also produced modest increases in the responses of 10 AMH-fibers to noxious heat (Figures 4D and E). The effect of XE991 was delayed in 6/10 fibers, the peak firing response occurring at 10-15 min after exposure. $3 \mu \mathrm{M}$ XE991 significantly $(P<0.05)$ increased the number of action potentials from $12.6 \pm$ 3.9 and $5.6 \pm 1.0$ in two controls, respectively, to a peak response of $46.8 \pm 8$.

In contrast to the sensitizing effect of XE991 on A $\delta$-fiber responses to noxious heat, $3 \mu \mathrm{M}$ XE991 had negligible effect on the responses of 7 out of $8 \mathrm{C}$-fibers tested (Figure 5). Five minutes' exposure of the receptive fields of $5 \mathrm{C}$-fibers $(4 \mathrm{CMH}$, $1 \mathrm{CMCH})$ to $3 \mu \mathrm{M}$ XE991 had no significant effect compared with control $(P>0.05$; Figure $5 \mathbf{H})$. The mean number of action potentials per heat stimulation was $47 \pm 32.5$ and $35.2 \pm 23.0$ in two controls, respectively, and $35 \pm 24.4$ at $5 \mathrm{~min}$ after exposure to XE991. In 3/8 C-fibers (2 CMH, $1 \mathrm{CMCH}$ ), the receptive fields 


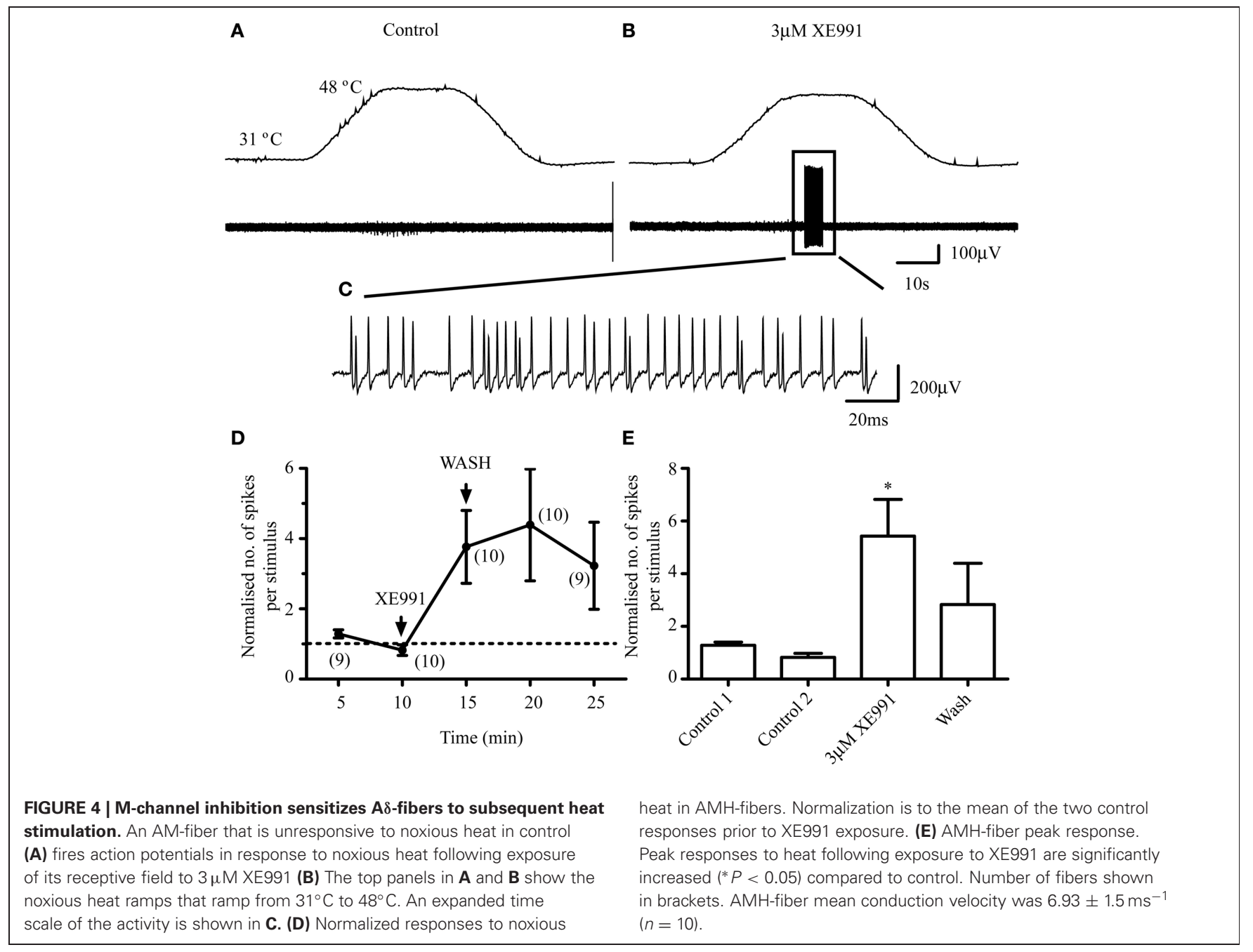

were exposed for an additional $5 \mathrm{~min}$. XE991 had no effect on the responses of the two $\mathrm{CMH}$-fibers but recruited the $\mathrm{CMCH}$-fiber during cooling from noxious heat so that it began firing robustly at $40.8^{\circ} \mathrm{C}$ compared with $31.2^{\circ} \mathrm{C}$ in control (data not shown). This latter observation was not investigated further due to the rarity of $\mathrm{CMCH}$-fibers in rat hairy skin (Zimmermann et al., 2009).

\section{M-CHANNELS IN UNMYELINATED FIBERS?}

The above results raise the question whether unmyelinated fibers in general possess functional M-channels. We tested this using rat vagus nerves in which around $80 \%$ of the fibers are unmyelinated (Soltanpour and Santer, 1996). As shown in Figure 6A, retigabine $(10 \mu \mathrm{M})$ consistently hyperpolarized the nerve in the central chamber with respect to the reference electrode in the distal chamber (mean axonal polarization $=0.38 \pm 0.022 \mathrm{mV}, n=33$ ). This was significantly reduced by $30 \mu \mathrm{M}$ linopirdine (from $0.42 \pm$ 0.04 to $0.19 \pm 0.02 ; n=11, P<0.005$ ) or by $10 \mu \mathrm{M} \mathrm{XE991} \mathrm{(from}$ $0.33 \pm 0.03$ to $0.09 \pm 0.02 \mathrm{mV} ; n=7, P<0.01$ ) (Figure 6C), showing that it resulted from increased M-channel activity. Although the nerve contains both $\mathrm{A}$ and $\mathrm{C}$ fibers, the hyperpolarization was generated, at least in part, in the C-fibers, since the evoked C-fiber compound action potential was enhanced and the post-spike hyperpolarization reduced (Figure 6B), as expected were the membrane potential shifted nearer to $E_{K}$. However, as is apparent from Figure 6A, linopirdine did not materially increase the resting demarcation potential, i.e., it did not depolarize the C-fibers. Thus, the maximum increases in demarcation potential produced by $10-30 \mu \mathrm{M}$ linopirdine and $10 \mu \mathrm{M}$ XE991 were only $0.071 \pm 0.015 \mathrm{mV}(n=10)$ and $0.048 \pm 0.016 \mathrm{mV}(n=6)$, respectively. Further, since all these preparations had previously been exposed to retigabine, it is likely that even this small response was due to block of residual retigabine-enhanced M-currents, not unmodified currents.

\section{In vivo EVIDENCE FOR PERIPHERAL M-CHANNEL MODULATION OF SENSORY FUNCTION}

In order to further characterize the physiological role of M-channels in peripheral sensory terminals, extracellular action potentials were recorded from deep DH neurons with receptive fields on the plantar hindpaw. These neurons were characterized as wide dynamic range cells (WDR), in that they gave a graded response to a wide range of innocuous and noxious mechanical and thermal stimuli, exhibited windup, and were located in 


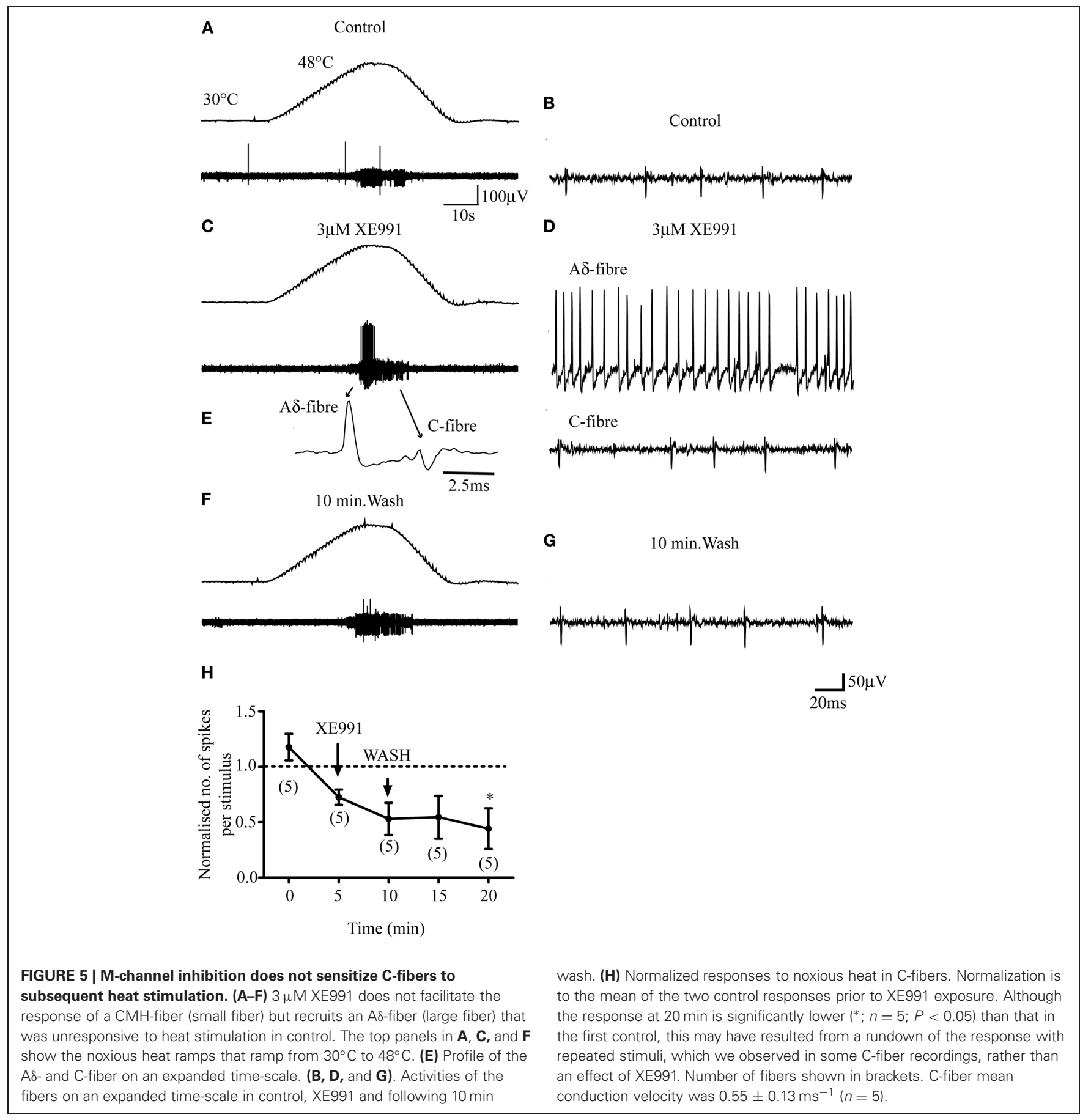

laminae V-VI of the DH, as previously described (e.g., Bee et al., 2011).

Intraplantar XE991 $(200 \mu \mathrm{M})$ had no significant effect on electrically-evoked responses, where stimulation is delivered directly to peripheral nerve endings, bypassing mechanical and thermal transduction mechanisms (Figure 7 panels $\mathbf{A}$ and $\mathbf{B}$, paired $t$-test of predose baseline against maximum change in post-dose hour of recording).

Spinal WDR cell responses to von Frey hairs applied to the hindpaw receptive field were selectively potentiated. The

responses to 6, 8, and $15 \mathrm{~g}$ von Frey hairs were significantly potentiated, while responses to the upper range of mechanical stimuli and to innocuous brush were not significantly altered (Figure 7 panel C, with expanded view of low range von Frey responses and brush response in panels $\mathbf{D}$ and $\mathbf{E}$, respectively. Two-Way ANOVA with Bonferroni's multiple comparison post-test, $n=8$ WDR cells).

Contrastingly, spinal WDR cell responses to thermal stimuli (water jet applied to the hindpaw receptive field) were significantly potentiated in the higher thermal range (Figure 7 panel F, 

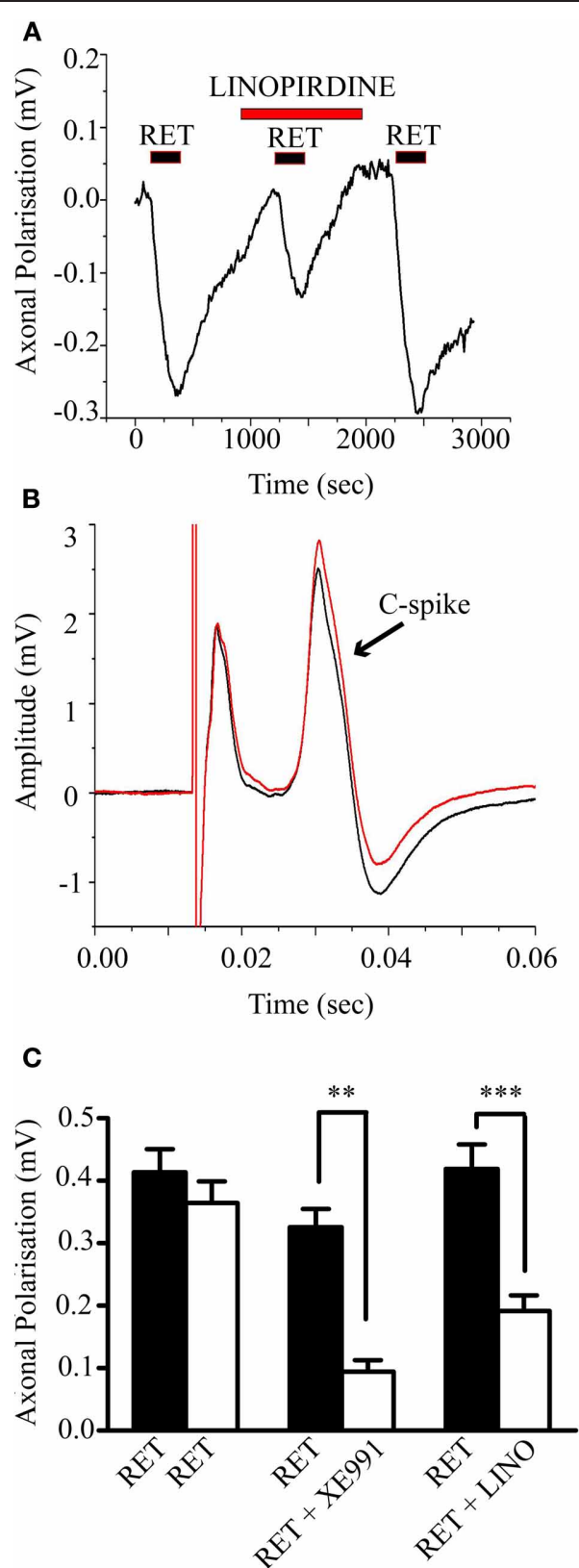

FIGURE 6 | Retigabine-induced a linopirdine-sensitive hyperpolarization of the rat vagus nerve with concomitant changes in the compound action potential generated by the unmyelinated axons (C-spike). (A) $10 \mu \mathrm{M}$ retigabine applied for $3 \mathrm{~min}$ induced a prolonged hyperpolarization of the vagus nerve that was reversibly inhibited by $30 \mu \mathrm{M}$ linopirdine. Hyperpolarization was recorded as the change in surface demarcation potential between the central drug-perfused chamber and the distal chamber containing the cut nerve ending. (B) The C-spike compound action potential amplitude (second deflection) was increased and the spike after-hyperpolarization was decreased by retigabine (red trace). Data has been baseline subtracted for ease of comparison. (C) Mean ( \pm SEM) demarcation potential changes produced by retigabine. The retigabine-induced hyperpolarization (black columns) was reproducible when reapplied after $1 \mathrm{~h}$ (first pair, open column; $n=8$ ) and significantly inhibited when the second application was preceded by $10 \mu \mathrm{M}$ XE991 (second pair, $n=7$; ** $P<0.01$ ) or $30 \mu \mathrm{M}$ linopirdine (third pair, $\left.n=11^{* * *} P<0.005\right)$ $48^{\circ} \mathrm{C}$; Two-Way ANOVA with Bonferroni’s multiple comparison post-test, $n=8 \mathrm{WDR}$ cells).

No consistent effect was seen on repetitive firing immediately following application of natural stimuli. No spontaneous firing of spinal WDR cells was observed following intraplantar XE991. In contrast to the effects of XE991 delivered intraplantar, central XE991 applied directly to the spinal cord (as doses of 1 or $10 \mu \mathrm{g}$ in $50 \mu \mathrm{l}$ saline) did not cause significant alteration in WDR cellevoked responses (data not shown).

These in vivo results corroborate the in vitro studies indicating a role for M-channels in physiological modulation of small afferent fibers, particularly A $\delta$-fibers, and extend this modulation to certain mechanosensitive as well as thermosensitive fibers.

\section{DISCUSSION}

In these experiments we have shown the presence of an M-channel subunit in sensory nerve endings by immunohistochemistry and have demonstrated a role for M-channels in the modulation of peripheral sensory transmission by both in vitro and in vivo electrophysiological preparations.

Immunohistochemistry has revealed the presence of Kv7.2 channel protein in some (but not all) sensory nerve endings in rat paw skin. Staining was observed in both myelinated and unmyelinated fibers, and in both nociceptive (TRPV1-staining) and non-nociceptive fibers. This accords with previous observations showing Kv7.2 immunoreactivity in both small and large neurons in the DRG (Passmore et al., 2003). Some of the DRG neurons also stained for Kv7.3 and Kv7.5 (Passmore et al., 2003). We have not tested for these in the present experiments but it has recently been reported that Kv7.5 is the predominant immunoreactive M-channel subunit in small sensory neurons and in vagal C-fibers, with Kv7.2 being restricted to vagal myelinated fibers where immunoreactivity was concentrated at nodes of Ranvier (King and Scherer, 2012). Interestingly, the converse appear to hold for baroceptor afferents, with Kv7.2 in the unmyelinated and small myelinated fibers and Kv7.5 in the larger afferents (Wladyka et al., 2008). Kv7.2 immunoreactivity has also been identified in sciatic nerve nodes of Ranvier (Schwarz et al., 2006) and at nerve-end neuromas in axotomised saphenous nerves (Roza et al., 2011). In contrast, $M$-channels in the peripheral endings of cutaneous rapidly-adapting mechanoreceptor afferents are composed of Kv7.4 subunits (Heidenreich et al., 2011).

Irrespective of the particular subunit involved, we have provided clear evidence for the presence of functional $\mathrm{M}$-channels in nociceptive afferent fiber endings in the skin of the rat's paw. Thus, the M-channel enhancer retigabine applied directly to the peripheral nerve endings consistently reduced the responses of A $\delta$ afferent fibers to noxious heat and this effect (where tested) was reversed by the M-channel blocker XE991. Retigabine also reduced thermal responses of afferent $\mathrm{C}$-fibers, though to a lesser extent. Lang et al. (2008) have also shown that retigabine reduces the electrical excitability of C-fibers in human sural nerve. Using different (mechanical and inflammatory chemical) stimuli, Roza and Lopez-Garcia (2008) reported that retigabine could reduce Cand $\mathrm{A} \delta$-fiber responses of neuromatose skin afferents but could observe no clear effect on intact fibers. Differences from the present experiments may stem from the different stimuli used, 


\section{Electrically Evoked Responses}

A

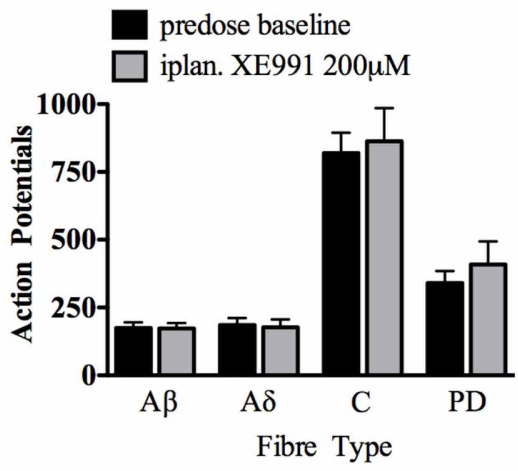

B

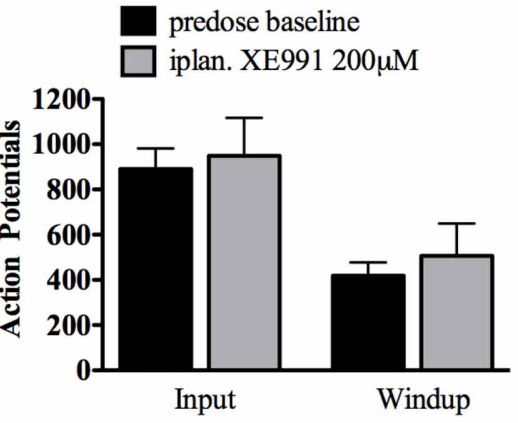

Static Mechanically Evoked Responses

c

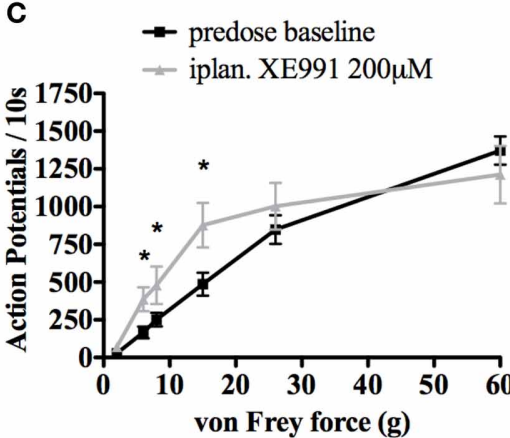

Dynamic Mechanically Evoked Responses

predose baseline

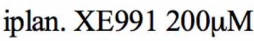

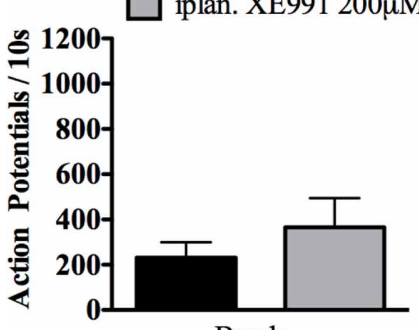

Brush

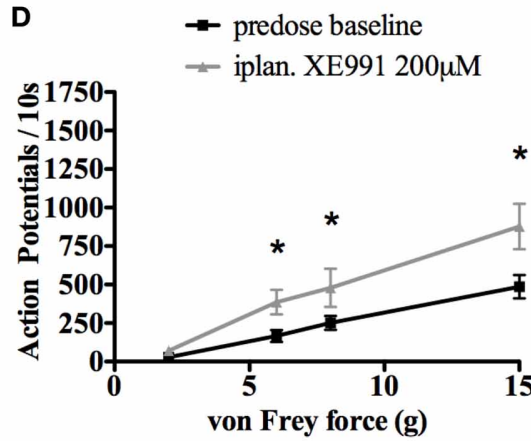

Thermally Evoked Responses

$\mathbf{F}$

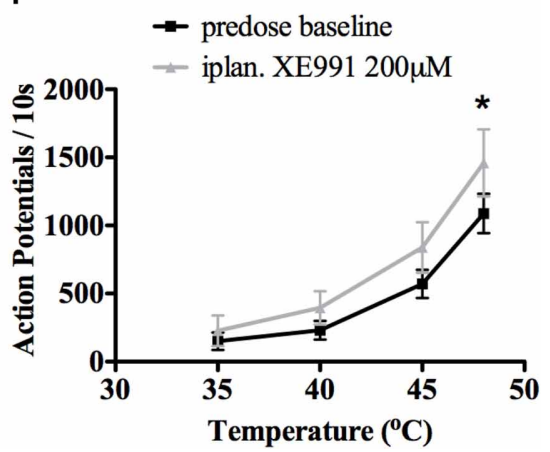

responses in (D) and high range thermally evoked responses (F) were all potentiated (for electrical stimuli: paired $t$-test of pre- vs. greatest change post-dose values; for natural stimuli: Two-Way ANOVA with Bonferroni's post-test of pre- vs. post-dose responses, ${ }^{*} P<0.05, n=8$ WDR cells). See Methods for technical details. implying perhaps a different fiber-population and/or a species difference.

These effects of retigabine confirm the presence of M-channels in nociceptive sensory nerve endings but do not show whether they affect normal physiological responses to nociceptor stimulation. This is better inferred from the effect of blocking the
M-channels. In the in vitro skin-saphenous nerve preparation we show that the M-channel blocker XE991 consistently and substantially enhanced the A $\delta$-fiber response to noxious thermal stimulation without clearly affecting the C-fiber response. Despite a low number of observations, a similar facilitation of A $\delta$-fiber responses to mechanical stimulation after exposure to 
XE991 has been previously reported (Roza and Lopez-Garcia, 2008). Further, in in vivo tests we found that intraplantar injection of XE991 selectively enhanced the response of deep DH neurons to peripheral mid-range mechanical and higher-range $\left(48^{\circ} \mathrm{C}\right)$ thermal stimuli.

While the lightest mechanical stimuli are likely to recruit mainly low threshold $A \beta$ fibers, and the most noxious 26 and $60 \mathrm{~g}$ von Frey hairs are likely to recruit high threshold C-fibers, A $\delta$-fibers are most likely responsible for transduction and transmission of mechanical stimuli in the middle range (Martin et al., 1987; Lynn and Shakhanbeh, 1988). It has recently been demonstrated in both rat and human that, somewhat counterintuitively, a cutaneously applied $\mathrm{CO}_{2}$ laser heat stimulus recruits first C-fibers, and then A $\delta$-fibers when its intensity is increased (Sikandar, personal communication; Mouraux et al., 2011). It is, therefore, likely that the potentiation of mid-range mechanical and upper range thermal-evoked responses by XE991 would be consistent with localization of M-channels primarily on A $\delta$-fiber peripheral terminals. This could be consistent with a preferential enhancement of peripheral A $\delta$-fiber discharges, also seen in the skin-saphenous nerve population. Although the concentration of XE991 used in these experiments was high $(200 \mu \mathrm{M})$, it matches that previously found to induce acute pain by intraplantar injection (Linley et al., 2008); indeed, our findings provide a clear rationale for the observation of enhanced behavioral responses. Interestingly, the spectrum of effects of XE991 in vivo partially resemble the polymodal effects seen in TREK1 gene-deleted mice (Alloui et al., 2006); like Kv7.2 (Rose et al., 2011), TREK-1 is strongly expressed in TRPV1-containing sensory neurons, so might co-localize with Kv7.2 in sensory nerve endings.

How might $\mathrm{M}$-channels regulate sensory nerve activation by noxious stimuli? One possibility is simply that sufficient M-channels are open at rest as to generate a component of the "leak" conductance. This may slightly hyperpolarize the nerve ending but more significantly add a shunt conductance that would reduce the amplitude of the nociceptor generator potential and raise the threshold for action potential initiation. The voltage-sensitivity of the channels would then enhance their effect during sensory nerve activation. This conductance shunt has been suggested to be responsible for the effect of Kv7.4 channels on cutaneous rapidly adapting mechanoreceptor discharges. Here the channels are localized to the unmyelinated terminals of myelinated fibers in Meissner's corpuscles and in the hair follicles, and exert a strong effect in attenuating low-frequency mechanosensory discharges (Heidenreich et al., 2011).

If the effect of M-channels on nociceptor afferent discharges results from a similar conductance-shunting effect at the unmyelinated fiber terminals, why then should the A $\delta$-fiber discharges be more sensitive than the C-fiber discharges to XE991? One possibility is that it relates to the different Kv7 subunits reported to be present in the two fiber types (Kv7.5 in unmyelinated afferents versus Kv7.2 in myelinated afferents: King and Scherer, 2012), since the former appear rather less sensitive to XE991 (IC 50 against Kv7.5 = $65 \mu \mathrm{M}$; Schroeder et al., 2000) than other Kv7 channels $\left(\mathrm{IC}_{50}\right.$ against $\mathrm{Kv7.2}=0.71 \mu \mathrm{M}$ and $\mathrm{Kv7.2} / 7.3=$ $0.6 \mu \mathrm{M}$, Wang et al., 1998) [although these have not been directly compared in the same set of experiments]. However, this seems unlikely, because the effects of retigabine on C-fiber excitability (Lang et al., 2008) and membrane potential (this paper, Figure 6C) were blocked by $10 \mu \mathrm{M}$ XE991.

An alternative explanation might be that the M-channels are not, in fact, very active at or near the resting potential in the unmyelinated terminals of nociceptor afferents. Thus, while there is evidence that $\mathrm{M}$-channels are active at resting potential in small sensory neurons (where their block produces a membrane depolarization and increases action potential discharges during depolarizing current injections: Passmore et al., 2003; Wladyka and Kunze, 2006; Wladyka et al., 2008; Liu et al., 2010) there is no direct evidence that this is so in peripheral nerve terminals. Indeed the excitability of sensory C-fibers in the sural nerve is not changed by XE991 (Lang et al., 2008), nor were vagal C-fibers substantially depolarized by XE991 or linopirdine, even though they were strongly hyperpolarized by retigabine and this hyperpolarization blocked by XE991 (present paper). Thus, M-channels were clearly present but normally inactive. Perhaps peripheral nociceptor terminals and unmyelinated fibers are maintained at a membrane potential negative to the activation threshold for the M-channels, for example, by electrogenic Na-pump activity (Rang and Ritchie, 1968) or by current flow through K2P "leak" channels or $\mathrm{Na}^{+}$-activated $\mathrm{K}_{\mathrm{Na}}$ (Slo2) channels. Both of the latter have been reported in sensory neurons (K2P: Plant, 2012; Marsh et al., 2012; $\mathrm{K}_{\mathrm{Na}}$ : Tamsett et al., 2009; Nuwer et al., 2010; see also Alloui et al., 2006; referred to above), though whether these channels are active in terminals or unmyelinated fibers is not yet clear.

In contrast, the excitability of myelinated fibers in peripheral nerves clearly is increased by blocking M-channels (Schwarz et al., 2006; Sittl et al., 2010). Hence, we propose that the greater sensitivity of the A $\delta$-fibers to XE991 results from the fact that, in peripheral myelinated fibers, the $\mathrm{M}$-channels are primarily expressed at nodes of Ranvier (Schwarz et al., 2006; King and Scherer, 2012), and also perhaps at the site of action potential initiation (Pan et al., 2006). At such loci, they would have a profound effect on the threshold for action potential generation and on the ability of the fibers to sustain trains of action potentials. Thus, inhibition of M-channel function at the axon initial segment of hippocampal neurons substantially lowers the action potential threshold, increases the firing frequency during neuron depolarization and can even induce spontaneous firing (Shah et al., 2008)—in total, very like the effects of XE991 on A $\delta$-fiber activity.

Then how might retigabine inhibit sensory discharges under circumstances where XE991 has no effect? The main effect of retigabine is to produce a hyperpolarizing shift of some $20 \mathrm{mV}$ or more in the voltage-dependence for M-channel activation (Tatulian et al., 2001). Hence, so long as M-channels were present, even if they were not normally very active at rest, they would now become so, and would produce a large hyperpolarization and large increase in the conductance shunt. Thus, even though C-fiber excitability is not normally enhanced by XE991, their excitability is strongly reduced by retigabine (Lang et al., 2008); and primary afferent fibers unresponsive to XE991 are strongly hyperpolarized by retigabine 
(Rivera-Arconada and Lopez-Garcia, 2006). In this connection, we have also noted a similar discrepancy between the effects of XE991 and retigabine with respect to the responses of $\mathrm{DH}$ neurons to XE991 and retigabine when applied to the spinal cord: whereas spinal cord application of retigabine strongly reduced nociceptive responses (Passmore et al., 2003), a similar application of XE991 in the present study had no effect. Thus, retigabine can attenuate nociceptive and other forms of hyper-excitability by enhancing $\mathrm{M}$-channel activity, even at sites where the channels do not normally have a very prominent physiological role.

The present data thus demonstrate an important physiological contribution of $\mathrm{M}$-channels to sensory function in the peripheral terminals of $A \delta$-fibers. They also suggest that these peripheral M-channels might be targeted by locally applied agents for the control of pain.

\section{AUTHOR CONTRIBUTIONS}

Gayle M. Passmore, Anthony H. Dickenson, and David A. Brown initiated the study; Gayle M. Passmore completed the

\section{REFERENCES}

Adams, P. R., Brown, D. A., and Constanti, A. (1982). Pharmacological inhibition of the M-current. J. Physiol. 332, 223-262.

Aiken, S. P., Lampe, B. J., Murphy, P. A., and Brown, B. S. (1995). Reduction of spike frequency adaptation and blockade of $\mathrm{M}$ current in rat CAl pyramidal neurones by linopirdine (DuP 996), a neurotransmitter release enhancer. Br. J. Pharmacol. 115, 1163-1168.

Alloui, A., Zimmermann, K., Mamet, J., Duprat, F., Noël, J., Chemin, J., Guy, N., Blondeau, N., Voilley, N., Rubat-Coudert, C., Borsotto, M., Romey, G., Heurteaux, C., Reeh, P., Eschalier, A., and Lazdunski, M. (2006). TREK-1, a $\mathrm{K} \pm$ channel involved in polymodal pain perception. EMBO J. 25, 2368-2376.

Bee, L. A., Bannister, K., Rahman, W., and Dickenson, A. H. (2011). Mu-opioid and noradrenergic $\alpha(2)$ adrenoceptor contributions to the effects of tapentadol on spinal electrophysiological measures of nociception in nerve-injured rats. Pain 152, 131-139.

Brown, D. A. (1988). "M currents", in Ion Channels, ed. T. Narahashi (New York, NY: Plenum Press), 55-94.

Brown, D. A., and Constanti, A. (1980). Intracellular observations on the effects of muscarinic agonists on rat sympathetic neurones. $B r$. J. Pharmacol. 70, 593-608.

Brown, D. A., and Passmore, G. M. (2009). Neural KCNQ (Kv7) channels. Br. J. Pharmacol. 156, 1185-1195.

Cuevas, J., Harper, A. A., Trequattrini, C., and Adams, D. J. (1997). Passive and active membrane properties of isolated rat intracardiac neurons: regulation by $\mathrm{H}-$ and M-currents. J. Neurophysiol. 78, 1890-1902.

$\mathrm{Du}$, J., Koltzenburg, M., and Carlton, S. M. (2001). Glutamate-induced excitation and sensitization of nociceptors in rat glabrous skin. Pain 89, 187-198.

Elmes, S. J., Millns, P. J., Smart, D., Kendall, D. A., and Chapman, V. (2004). Evidence for biological effects of exogenous LPA on rat primary afferent and spinal cord neurons. Brain Res. 1022, 205-213.

Hadley, J. K., Passmore, G. M., Tatulian, L., Al-Qatari, M., Ye, F., Wickenden, A. D., and Brown, D. A. (2003). Stoichiometry of expressed KCNQ2/KCNQ3 potassium channels and subunit composition of native ganglionic $M$ channels deduced from block by tetraethylammonium. J. Neurosci. 23, 5012-5019.

Heidenreich, M., Lechner, S. G., Vardanyan, V., Wetzel, C., Cremers, C. W., De Leenheer, E. M., Aránguez, G., Moreno-Pelayo, M. Á., Jentsch, T. J., and Lewin, G. R. (2011). KCNQ4 K(+) channels tune mechanoreceptors for normal touch sensation in mouse and man. Nat. Neurosci. 15, 138-145.

King, C. H., and Scherer, S. S. (2012). Kv7.5 is the primary Kv7 subunit expressed in C-fibers. J. Comp. Neurol. 520, 1940-1950.

experiments on the in vitro skin-nerve preparation; Joanne M. Reilly completed the immunohistochemical work; Joanne M. Reilly, Vanessa N. Keasberry, and Stephen J. Marsh did the experiments on the vagus nerves; Matthew Thakur completed the in vivo dorsal horn recordings; all authors (led by Gayle M. Passmore) contributed to the writing of the paper.

\section{ACKNOWLEDGMENTS}

This work was supported by grants from the Medical Research Council (G0500194) and the Wellcome Trust-funded London Pain Consortium (083858). We thank Professor Stuart Bevan and Dr. Alyson Fox, former members of the Novartis Institute for Medical Sciences, for the skin-nerve apparatus and Sian Moss for her initial help with the technique. We also thank Professor Steve Hunt and his lab for their initial help with the skin sectioning, Daniel Ciantar for his help with the confocal imaging, Professor Peter Reeh for the calibrated aluminium von Frey hairs and Dr. Katarina Zimmermann and Dr. Jon Robbins for helpful discussions. Retigabine was supplied by Neurosearch (Ballerup, Denmark) via EU grant LSHM-CT-2004-503038.

Lang, P. M., Fleckenstein, J., Passmore, G. M., Brown, D. A., and Grafe, P. (2008). Retigabine reduces the excitability of unmyelinated peripheral human axons. Neuropharmacology 54, 1271-1278.

Linley, J. E., Rose, K., Patil, M., Robertson, B., Akopian, A. N., and Gamper, N. (2008). Inhibition of $M$ current in sensory neurons by exogenous proteases: a signalling pathway mediating inflammatory nociception. J. Neurosci. 28, 11240-11249.

Liu, B., Linley, J. E., Du, X., Zhang, X., Ooi, L., Zhang, H., and Gamper, N. (2010). The acute nociceptive signals induced by bradykinin in rat sensory neurons are mediated by inhibition of M-type $\mathrm{K} \pm$ channels and activation of $\mathrm{Ca} 2 \pm$-activated Cl- channels. J. Clin. Invest. 120, 1240-1252.

Lynn, B., and Shakhanbeh, J. (1988). Properties of A delta high threshold mechanoreceptors in the rat hairy and glabrous skin and their response to heat. Neurosci. Lett. 85 , 71-76.

Marsh, B., Acosta, C., Djouhri, L., and Lawson, S. N. (2012). Leak K(+) channel mRNAs in dorsal root ganglia: relation to inflammation and spontaneous pain behaviour. Mol. Cell. Neurosci. 49, 375-386.

Marsh, S. J., Stansfeld, C. E., Brown, D. A., Davey, R., and McCarthy, D. (1987). The mechanism of action of capsaicin on sensory C-type neurons and their axons in vitro. Neuroscience 23, 275-289.

Martin, H. A., Basbaum, A. I., Kwiat, G. C., Goetzl, E. J., and Levine,
J. D. (1987). Leukotriene and prostaglandin sensitization of cutaneous high-threshold C- and A-delta mechanonociceptors in the hairy skin of rat hindlimbs. Neuroscience 22, 651-659.

Mouraux, A., Iannetti, G. D., Colon, E., Nozaradan, S., Legrain, V., and Plaghki, L. (2011). Nociceptive steady-state evoked potentials elicited by rapid periodic thermal stimulation of cutaneous nociceptors. J. Neurosci. 31, 6079-6087.

Mucha, M., Ooi, L., Linley, J. E., Mordaka, P., Dalle, C., Robertson, B., Gamper, N., and Wood, I. C. (2010). Transcriptional control of KCNQ channel genes and the regulation of neuronal excitability. J. Neurosci. 30, 13235-13245.

Nuwer, M. O., Picchione, K. E., and Bhattacharjee, A. (2010). PKAinduced internalization of slack $\mathrm{KNa}$ channels produces dorsal root ganglion neuron hyperexcitability. J. Neurosci. 30,14165-14172.

Pan, Z., Kao, T., Horvath, Z., Lemos, J., Sul, J. Y., Cranstoun, S. D. Bennett, V., Scherer, S. S., and Cooper, E. C. (2006). A common ankyrin-G-based mechanism retains $\mathrm{KCNQ}$ and $\mathrm{NaV}$ channels at electrically active domains of the axon. J. Neurosci. 26, 2599-2613.

Passmore, G. M., and Brown, D. A (2007). Effects of M-channel modulators on peripheral excitability in rat hairy skin. Soc. Neurosci. Abstr. 681.8.

Passmore, G. M., Selyanko, A. A., Mistry, M., Al-Qatari, M., Marsh, S. J., Matthews, E. A., Dickenson, A. 
H., Brown, T. A., Burbidge, S. A., Main, M., and Brown, D. A. (2003). KCNQ/M currents in sensory neurons: significance for pain therapy. J. Neurosci. 23, 7227-7236.

Peters, H. C., Hu, H., Pongs, O., Storm, J. F., and Isbrandt, D. (2005). Conditional transgenic suppression of $\mathrm{M}$ channels in mouse brain reveals functions in neuronal excitability, resonance and behavior. Nat. Neurosci. 8, 51-60.

Plant, L. D. (2012). A role for K2P channels in the operation of somatosensory nociceptors. Front. Mol. Neurosci. 5:21. doi: 10.3389/fnmol.2012.00021

Rang, H. P., and Ritchie, J. M. (1968). On the electrogenic sodium pump in mammalian non-myelinated nerve fibres and its activation by various external cations. J. Physiol. 196, 183-221.

Reeh, P. W. (1986). Sensory receptors in mammalian skin in an in vitro preparation. Neurosci. Lett. 66, 141-146.

Reilly, J. M., Passmore, G. M., and Brown, D. A. (2008). M-/Kv7 channel localisation and function in peripheral afferent fibres of isolated rat skin. FENS Abstr. 4, 078.23.

Rivera-Arconada, I., and Lopez-Garcia, J. A. (2005). Effects of M-current modulators on the excitability of immature rat spinal sensory and motor neurons. Eur. J. Neurosci. 22, 3091-3098.

Rivera-Arconada, I., and Lopez-Garcia, J. A. (2006). Retigabine-induced population primary afferent hyperpolarisation in vitro. Neuropharmacology 51, 756-763.

Rose, K., Ooi, L., Dalle, C., Robertson, B., Wood, I. C., and Gamper, N.
(2011). Transcriptional repression of the M channel subunit Kv7.2 in chronic nerve injury. Pain 152, 742-754.

Roza, C., and Lopez-Garcia, J. A. (2008). Retigabine, the specific KCNQ channel opener, blocks ectopic discharges in axotomized sensory fibres. Pain 138, 537-545.

Roza, C., Castillejo, S., and LopezGarcía, J. A. (2011). Accumulation of Kv7.2 channels in putative ectopic transduction zones of mice nerve-end neuromas. Mol. Pain 7, 58-64.

Schroeder, B. C., Hechenberger, M., Weinreich, F., Kubisch, C., and Jentsch, T. J. (2000). KCNQ5, a novel potassium channel broadly expressed in brain, mediates $\mathrm{M}$ type currents. J. Biol. Chem. 275, 24089-24095.

Schwarz, J. R., Glassmeier, G., Cooper, E. C., Kao, T. C., Nodera, H., Tabuena, D., Kaji, R., and Bostock, H. (2006). KCNQ channels mediate IKs, a slow $\mathrm{K}+$ current regulating excitability in the rat node of Ranvier. J. Physiol. 573, 17-34.

Shah, M. M., Migliore, M., Valencia, I., Cooper, E. C., and Brown, D. A. (2008). Functional significance of axonal Kv7 channels in hippocampal pyramidal neurons. Proc. Natl. Acad. Sci. U.S.A. 105 7869-7874.

Shen, W., Hamilton, S. E., Nathanson, N. M., and Surmeier, D. J. (2005). Cholinergic suppression of KCNQ channel currents enhances excitability of striatal medium spiny neurons. J. Neurosci. 25, 7449-7458.

Sittl, R., Carr, R. W., Schwarz, J. R., and Grafe, P. (2010). The Kv7 potassium channel activator flupirtine affects clinical excitability parameters of myelinated axons in isolated rat sural nerve. J. Peripher. Nerv. Syst. 15, 63-72.

Soltanpour, N., and Santer, R. M. (1996). Preservation of the cervical vagus nerve in aged rats: morphometric and enzyme histochemical evidence. J. Auton. Nerv. Syst. 60, 93-101.

Tamsett, T. J., Picchione, K. E., and Bhattacharjee, A. (2009). $\mathrm{NAD}^{+}$ activates $\mathrm{K}_{\mathrm{Na}}$ channels in dorsal root ganglion neurons. J. Neurosci. 29, 5127-5134.

Tatulian, L., Delmas, P., Abogadie, F., and Brown, D. A. (2001). Activation of expressed KCNQ potassium currents and native neuronal M-type potassium currents by the anti-convulsant drug retigabine. J. Neurosci. 21, 5535-5545.

Urch, C. E., and Dickenson, A. H (2003). In vivo single unit extracellular recordings from spinal cord neurons of rats. Brain Res. Brain Res. Protoc. 12, 26-34.

Wang, H. S., Pan, Z., Shi, W., Brown, B. S., Wymore, R. S., Cohen, I. S., Dixon, J. E., and McKinnon, D. (1998). KCNQ2 and KCNQ3 potassium channel subunits: molecular correlates of the M-channel. Science 282, 1890-1893.

Wladyka, C. L., Feng, B., Glazebrook, P. A., Schild, J. H., and Kunze, D. L. (2008). The KCNQ/M-current modulates arterial baroreceptor function at the sensory terminal in rats. J. Physiol. 586, 795-802.

Wladyka, C. L., and Kunze, D. L. (2006). KCNQ/M-currents contribute to the resting membrane potential in rat visceral sensory neurons. J. Physiol. 575, 175-189.

Zaika, O., Lara, L. S., Gamper, N., Hilgemann, D. W., Jaffe, D. B., and Shapiro, M. S. (2006). Angiotensin-II regulates neuronal excitability via phosphatidylinositol 4,5-bisphosphate-dependent modulation of Kv7 (M-type) K+ channels. J. Physiol. 575, 49-67.

Zimmermann, K., Hein, A., Hager, U., Kaczmarek, J. S., Turnquist, B. P., Clapham, D. E., and Reeh, P. W. (2009). Phenotyping sensory nerve endings in vitro in the mouse. Nat. Protoc. 4, 174-196.

Conflict of Interest Statement: The authors declare that the research was conducted in the absence of any commercial or financial relationships that could be construed as a potential conflict of interest.

Received: 21 February 2012; accepted: 24 April 2012; published online: 14 May 2012.

Citation: Passmore GM, Reilly JM, Thakur M, Keasberry VN, Marsh SJ, Dickenson AH and Brown DA (2012) Functional significance of $M$-type potassium channels in nociceptive cutaneous sensory endings. Front. Mol. Neurosci. 5:63. doi: 10.3389/fnmol.2012.00063

Copyright (C) 2012 Passmore, Reilly, Thakur, Keasberry, Marsh, Dickenson and Brown. This is an open-access article distributed under the terms of the Creative Commons Attribution Non Commercial License, which permits non-commercial use, distribution, and reproduction in other forums, provided the original authors and source are credited. 